\title{
Assessing the Extended In-Use Stability of the Infliximab Biosimilar PF-06438179/GP1111 Following Preparation for Intravenous Infusion
}

\author{
Maja Vimpolsek ${ }^{1} \cdot$ Marie Gottar-Guillier $^{2} \cdot$ Emmanuel Rossy $^{3}$
}

Published online: 27 February 2019

(c) The Author(s) 2019

\begin{abstract}
Objective PF-06438179/GP1111 (PF-SZ-IFX) is an infliximab biosimilar. We evaluated the extended in-use physicochemical and biological stability of PF-SZ-IFX upon preparation for intravenous infusion.

Methods Two batches of PF-SZ-IFX were reconstituted to a concentration of $10 \mathrm{mg} / \mathrm{mL}$ and subsequently diluted to 0.4 and $4.0 \mathrm{mg} / \mathrm{mL}$, representing the clinically relevant range for intravenous infusion. Dilution was performed in polyethylene saline infusion bags, which are commonly used in clinical practice. To simulate product handling under worst-case conditions, reconstituted solutions were stored for up to 30 days at $5 \pm 3{ }^{\circ} \mathrm{C}$ and up to 14 days at $25 \pm 2{ }^{\circ} \mathrm{C}(60 \pm 5 \%$ relative humidity); diluted solutions were stored for up to 30 days under the same sets of conditions. Physicochemical and biological stability were evaluated according to $\mathrm{pH}$, osmolality, appearance, particulate content, protein concentration, proportions of molecular weight variants and charge variants and potency. Standard and state-of-the-art analytical techniques were employed, including imaged isoelectric focusing, size exclusion chromatography, reducing sodium dodecyl sulphate capillary electrophoresis and functional cell-based bioassay. Results Across batches and concentrations of PF-SZ-IFX, all parameters resided within the predefined acceptance criteria, including $\mathrm{pH}$, osmolality, particulate content, clarity, protein concentration, molecular weight variants, charge variants and potency, for up to 30 days under both storage conditions tested (up to 14 days for reconstituted samples stored at $25 \pm 2{ }^{\circ} \mathrm{C}$ ). Conclusions Physicochemical and biological analyses demonstrated that the infliximab biosimilar PF-SZ-IFX was not affected by extended storage of the diluted preparations used for intravenous infusion.
\end{abstract}

\section{Key Points}

Following reconstitution to a concentration of $10 \mathrm{mg} /$ $\mathrm{mL}$, the in-use physicochemical and biological stability of the infliximab biosimilar PF-06438179/GP1111 (PFSZ-IFX) was maintained for 30 days at $5 \pm 3{ }^{\circ} \mathrm{C}$ and for 14 days at $25 \pm 2{ }^{\circ} \mathrm{C}(60 \pm 5 \%$ relative humidity $)$.

Following dilution in polyethylene saline infusion bags to 0.4 and $4.0 \mathrm{mg} / \mathrm{mL}$, the stability of PF-SZ-IFX was maintained for up to 30 days at $5 \pm 3{ }^{\circ} \mathrm{C}$ or $25 \pm 2{ }^{\circ} \mathrm{C}$ ( $60 \pm 5 \%$ relative humidity).

The infliximab biosimilar PF-SZ-IFX was not affected by reconstitution, dilution and/or storage conditions required for intravenous infusion in clinical practice.

Maja Vimpolsek

maja.vimpolsek@novartis.com

1 Biopharmaceutical Product and Process Development, Drug Product Analytics, Novartis, Lek d. d., Menges, Slovenia

\section{Introduction}

Infliximab, a chimeric immunoglobulin G1 monoclonal antibody that binds human tumour necrosis factor (TNF)- $\alpha$, is a biologic medicine used for the treatment of immune-mediated inflammatory diseases $[1,2]$. There is a strong interest in the development of biosimilars of marketed biologics, including infliximab, because of their potential for reducing treatment costs and increasing patient access to these important medicines [3]. Biosimilars and their reference medicines are required to contain matching active ingredients and exhibit similarity upon extensive physicochemical, biological and comparative clinical evaluations [4-6].

2 Biopharmaceutical Product and Process Development, Analytical Development and Characterization, Novartis, Basel, Switzerland

3 Biopharmaceutical Product and Process Development, Analytical Characterization, Novartis, Hexal AG, Oberhaching, Germany 
PF-06438179/GP1111 (PF-SZ-IFX) is an infliximab biosimilar. Structural, functional and in vivo nonclinical studies demonstrated that PF-SZ-IFX structurally and functionally matched reference infliximab [7]. The pharmacokinetic profile of PF-SZ-IFX was comparable to that of reference infliximab in healthy individuals [8], and PFSZ-IFX and reference infliximab demonstrated similar efficacy, safety, immunogenicity and pharmacokinetics with or without dose escalation, in patients with moderateto-severe active rheumatoid arthritis on a background of methotrexate [9]. PF-SZ-IFX is approved for the treatment of rheumatoid arthritis, Crohn's disease, ulcerative colitis, ankylosing spondylitis, psoriatic arthritis and psoriasis in the EU (Zessly ${ }^{\circledR}$ ) [10], Japan (Infliximab Biosimilar 3) [11] and the USA (IXIFI $\left.{ }^{\circledR}\right)$ [12].

Similar to new medicinal products, biosimilars undergo extensive stability and in-use testing to ensure that product quality is robust to standard transport and storage conditions $[13,14]$. While these data are submitted to national health authorities to confirm that product specifications are met throughout the declared shelf life and immediate in-use handling, the stability testing requirements do not cover extended in-use handling (e.g. longer storage of the diluted medications before they are used for intravenous infusion) $[15,16]$. Extended in-use storage is the responsibility of the healthcare professional who prepares the medication, which should be done under appropriate aseptic working conditions. Confirmation and reporting of product stability under extended in-use conditions provides valuable assurance to these healthcare professionals that pharmaceutical safety and efficacy can be maintained under appropriate reformulation (i.e. aseptic) and handling/storage conditions [17].

The present study aimed to bridge an existing knowledge gap and provide healthcare professionals with information on the stability of the infliximab biosimilar PF-SZIFX under extended in-use conditions after dilution and reformulation of the medicine for intravenous infusion.

\section{Methods}

\subsection{Sample Preparation and Storage Conditions}

Vials of PF-SZ-IFX product were produced under aseptic conditions in line with good manufacturing practice (GMP). Reconstitution and dilution were performed in a GMP laboratory in full accordance with the instructions detailed in the summary of product characteristics [10]. Where appropriate, experiments were conducted in line with compendial methods as required by regulatory authorities and defined in the European Pharmacopeia (Eur Ph) [18], US Pharmacopeia (USP) [19] and Japanese Pharmacopeia (JP) [20].
A total of 55 PF-SZ-IFX vials (two representative batches: 'batch A' and 'batch B') were reconstituted with $10 \mathrm{~mL}$ of sterile water for injection (Omnipur, UK) using a BD Microlance 21-gauge needle (B. Braun, Germany) to obtain a theoretical concentration of $10 \mathrm{mg} / \mathrm{mL}$. Of these, 33 vials were reconstituted for storage at $5 \pm 3{ }^{\circ} \mathrm{C}$ for up to 30 days $(n=11)$ or $25 \pm 2{ }^{\circ} \mathrm{C}(60 \pm 5 \%$ relative humidity [RH]) for up to 14 days $(n=22)$, and 22 vials were reconstituted for dilution.

Vials of reconstituted antibody $(10 \mathrm{mg} / \mathrm{mL})$ were diluted in $250 \mathrm{~mL}$ isotonic $0.9 \% \mathrm{NaCl}$ bags made of polyethylene (B. Braun) to obtain two concentrations covering the administration range recommended for PF-SZ-IFX (approximately $0.4 \mathrm{mg} / \mathrm{mL}$ [one vial] and $4.0 \mathrm{mg} / \mathrm{mL}$ [ten vials]). One bag of each concentration was stored for up to 30 days at $5 \pm 3{ }^{\circ} \mathrm{C}$ and at $25 \pm 2{ }^{\circ} \mathrm{C}(60 \pm 5 \% \mathrm{RH})$.

At each pull point, reconstituted samples $(10 \mathrm{mg} / \mathrm{mL})$ were allowed to equilibrate to room temperature for approximately $1 \mathrm{~h}$, and diluted samples $(0.4$ and $4.0 \mathrm{mg} / \mathrm{mL})$ were allowed to equilibrate to room temperature for $1-2 \mathrm{~h}$. Aliquots were drawn from the infusion bags by sterile syringe and needle, and infusion bags were returned to storage immediately after sampling. Osmolality, $\mathrm{pH}$, appearance, particulate content (visible and sub-visible), protein concentration, charge heterogeneity, purity and potency were analysed using the methods outlined in the following sections.

\subsection{Osmolality, pH and Appearance (Colour of Solution and Clarity)}

Osmolality, $\mathrm{pH}$ and appearance were evaluated to demonstrate integrity of the solution upon reconstitution, dilution and storage under in-use conditions, as a change in the solution properties could potentially affect tolerability upon intravenous injection.

Osmolality was measured by freezing point depression on an Advanced Instruments 3320 osmometer according to Eur Ph 2.2.35 ('Osmolality'), USP 785 ('Osmolality and osmolarity') and JP 2.47 ('Osmolarity determination').

$\mathrm{pH}$ was determined using a calibrated Metrohm $\mathrm{pH}$ meter (Columbus, USA), according to Eur Ph 2.2.3 ('Potentiometric determination of pH'), USP 791 (' $\mathrm{pH}$ ') and JP 2.54 (' $\mathrm{pH}$ determination').

For colouration assessment, samples were transferred to glass test tubes with an external diameter of $12 \mathrm{~mm}$. The degree of coloration was assessed by visual inspection according to Eur Ph 2.2.2 ('Degree of coloration of liquids').

For clarity assessment, turbidity measurements were performed instrumentally according to Eur Ph 2.2.1 ('Clarity and degree of opalescence liquids') using an HACH 2100AN turbidimeter (Hach Company, USA), which had been calibrated against certified turbidity standards. The results were expressed as nephelometric turbidity units (NTU). 
Table 1 Osmolality, pH and clarity for PF-SZ-IFX batches following in-use preparation for intravenous infusion

\begin{tabular}{|c|c|c|c|c|c|c|c|c|}
\hline \multirow[t]{2}{*}{ Batch } & \multirow{2}{*}{$\begin{array}{l}\text { Theoretical infliximab } \\
\text { conc. }(\mathrm{mg} / \mathrm{mL})\end{array}$} & \multirow[t]{2}{*}{ Time (days) } & \multicolumn{3}{|l|}{$5^{\circ} \mathrm{C}$} & \multicolumn{3}{|l|}{$25^{\circ} \mathrm{C} / 60 \% \mathrm{RH}$} \\
\hline & & & $\begin{array}{l}\text { Osmolality } \\
(\mathrm{mOsmol} / \mathrm{kg})\end{array}$ & $\mathrm{pH}$ & Clarity (NTU) & $\begin{array}{l}\text { Osmolality } \\
\text { (mOsmol/kg) }\end{array}$ & $\mathrm{pH}$ & Clarity (NTU) \\
\hline \multirow[t]{12}{*}{$\mathrm{A}$} & \multirow[t]{4}{*}{0.4} & 0 & 281 & 5.8 & 0.6 & 282 & 5.8 & 0.5 \\
\hline & & 7 & 294 & 5.4 & 0.4 & 290 & 5.7 & 0.3 \\
\hline & & 14 & 284 & 5.9 & 0.3 & 286 & 5.9 & 0.4 \\
\hline & & 30 & 284 & 5.8 & 0.9 & 281 & 5.9 & 0.4 \\
\hline & \multirow[t]{4}{*}{4.0} & 0 & 216 & 5.9 & 3.4 & 216 & 5.9 & 3.1 \\
\hline & & 7 & 222 & 5.9 & 3.2 & 231 & 5.9 & 3.3 \\
\hline & & 14 & 219 & 5.9 & 3.2 & 217 & 5.9 & 3.0 \\
\hline & & 30 & 218 & 5.9 & 3.2 & 217 & 5.9 & 3.2 \\
\hline & \multirow[t]{4}{*}{10} & 0 & 93 & 6.0 & 7.7 & 93 & 6.0 & 7.7 \\
\hline & & 7 & $\mathrm{nt}$ & $\mathrm{nt}$ & $\mathrm{nt}$ & 99 & 6.1 & 7.7 \\
\hline & & 14 & $\mathrm{nt}$ & nt & $\mathrm{nt}$ & 92 & 6.1 & 7.7 \\
\hline & & 30 & 90 & 6.1 & 7.4 & $\mathrm{nt}$ & $\mathrm{nt}$ & $\mathrm{nt}$ \\
\hline \multirow[t]{12}{*}{ B } & \multirow[t]{4}{*}{0.4} & 0 & 281 & 5.8 & 0.7 & 281 & 5.8 & 0.9 \\
\hline & & 7 & 290 & 5.5 & 0.5 & 289 & 5.6 & 0.4 \\
\hline & & 14 & 281 & 5.9 & 0.4 & 282 & 5.9 & 0.4 \\
\hline & & 30 & 283 & 5.8 & 0.3 & 282 & 5.9 & 0.5 \\
\hline & \multirow[t]{4}{*}{4.0} & 0 & 217 & 5.9 & 3.1 & 215 & 5.9 & 3.1 \\
\hline & & 7 & 223 & 5.9 & 3.2 & 220 & 5.9 & 3.2 \\
\hline & & 14 & 217 & 5.9 & 3.0 & 217 & 5.9 & 3.0 \\
\hline & & 30 & 219 & 5.9 & 3.4 & 217 & 5.9 & 3.2 \\
\hline & \multirow[t]{4}{*}{10} & 0 & 91 & 5.9 & 7.4 & 91 & 5.9 & 7.4 \\
\hline & & 7 & $\mathrm{nt}$ & $\mathrm{nt}$ & $\mathrm{nt}$ & 100 & 6.0 & 7.7 \\
\hline & & 14 & $\mathrm{nt}$ & $\mathrm{nt}$ & $\mathrm{nt}$ & 92 & 6.1 & 7.6 \\
\hline & & 30 & 93 & 6.1 & 7.5 & $\mathrm{nt}$ & $\mathrm{nt}$ & $\mathrm{nt}$ \\
\hline
\end{tabular}

Assessment of osmolality, $\mathrm{pH}$ and clarity in two PF-SZ-IFX batches reconstituted to $10 \mathrm{mg} / \mathrm{mL}$ and subsequently diluted in polyethylene saline solution infusion bags to 0.4 and $4.0 \mathrm{mg} / \mathrm{mL}$. Samples were stored at $5 \pm 3{ }^{\circ} \mathrm{C}$ or $25 \pm 2{ }^{\circ} \mathrm{C}(60 \pm 5 \% \mathrm{RH})$ for 7,14 or 30 days. Respective predefined acceptance criteria for osmolality/pH/clarity of 0.4 and $4.0 \mathrm{mg} / \mathrm{mL}$ dilutions, where $X$ is initial test result at T0: $X \pm 20 \mathrm{mOsmol} / \mathrm{kg}$, $\mathrm{pH}$ $X \pm 0.5, X \leq 8 \mathrm{NTU}$

$n t$ not tested, NTU nephelometric turbidity unit, $P F-S Z-I F X$ PF-06438179/GP1111, $R H$ relative humidity

\subsection{Particles}

The amount of sub-visible particles allowed in therapeutics is governed by USP 788 [21], with the goals being to prevent capillary occlusion from particles over $10 \mu \mathrm{m}$ that might be found in therapeutics delivered intravenously and to avoid potential safety concerns. Sub-visible particles were counted and sized by light obscuration according to Eur Ph 2.9.19 ('Particulate contamination: sub-visible particles'), USP 788 ('Particulate matter in injections') and JP 6.07 ('Insoluble particulate matter test for injections') on the HIAC 9703 + particle size counter (Beckman Coulter, USA). Sample volume was 10 vials $(10 \mathrm{mg} / \mathrm{mL})$ or $25 \mathrm{~mL}$ ( 0.4 and $4.0 \mathrm{mg} / \mathrm{mL}$ ), with four repetitions per sample. Samples were also inspected visually for particles.

\subsection{Protein Concentration}

Protein concentration was measured to evaluate whether adsorption of PF-SZ-IFX occurred under extended in-use conditions. Protein concentration was determined using a Perkin Elmer Lambda 35 ultraviolet (UV) spectrophotometer, calibrated following GMP standards and periodic qualification protocols by using tests predefined by the manufacturer. Samples were diluted gravimetrically and transferred to $1-\mathrm{cm}$ path length UV transparent quartz cuvettes (Hellma Analytics, Germany), with $0.9 \% \mathrm{NaCl}$ used as the blank solution for normalization purposes. Corrected absorbance ( $A_{\text {corr }}=A_{280 \mathrm{~nm}}-A_{320 \mathrm{~nm}}$ ) was determined and protein concentration calculated using the Beer-Lambert equation and a PF-SZ-IFX-specific extinction coefficient: 
Table 2 Light obscuration analysis of particulate contamination for PF-SZIFX batches following in-use preparation for intravenous infusion

\begin{tabular}{|c|c|c|c|c|c|c|}
\hline \multirow[t]{2}{*}{ Batch } & \multirow{2}{*}{$\begin{array}{l}\text { Theoretical inflixi- } \\
\text { mab conc. }(\mathrm{mg} / \mathrm{mL})\end{array}$} & \multirow[t]{2}{*}{ Time (days) } & \multicolumn{2}{|l|}{$5^{\circ} \mathrm{C}$} & \multicolumn{2}{|l|}{$25^{\circ} \mathrm{C} / 60 \% \mathrm{RH}$} \\
\hline & & & $\begin{array}{l}\text { Particle count } \\
\leq 10 \mu \mathrm{m}(n)\end{array}$ & $\begin{array}{l}\text { Particle count } \\
\leq 25 \mu \mathrm{m}(n)\end{array}$ & $\begin{array}{l}\text { Particle count } \\
\leq 10 \mu \mathrm{m}(n)\end{array}$ & $\begin{array}{l}\text { Particle } \\
\text { count } \leq \\
25 \mu \mathrm{m}(n)\end{array}$ \\
\hline \multirow[t]{12}{*}{ A } & \multirow[t]{4}{*}{0.4} & 0 & 233 & 15 & 1039 & 21 \\
\hline & & 7 & 631 & 27 & 1031 & 85 \\
\hline & & 14 & 37 & 1 & 1044 & 45 \\
\hline & & 30 & 1389 & 141 & 405 & 47 \\
\hline & \multirow[t]{4}{*}{4.0} & 0 & 945 & 32 & 1138 & 44 \\
\hline & & 7 & 623 & 16 & 1105 & 11 \\
\hline & & 14 & 479 & 13 & 1117 & 25 \\
\hline & & 30 & 135 & 5 & 238 & 12 \\
\hline & \multirow[t]{4}{*}{10} & 0 & 93 & 4 & 93 & 4 \\
\hline & & 7 & $\mathrm{nt}$ & nt & 368 & 9 \\
\hline & & 14 & $\mathrm{nt}$ & nt & 183 & 4 \\
\hline & & 30 & 638 & 5 & nt & $\mathrm{nt}$ \\
\hline \multirow[t]{12}{*}{ B } & \multirow[t]{4}{*}{0.4} & 0 & 359 & 73 & 2105 & 433 \\
\hline & & 7 & 383 & 29 & 255 & 14 \\
\hline & & 14 & 65 & 11 & 616 & 24 \\
\hline & & 30 & 293 & 29 & 498 & 37 \\
\hline & \multirow[t]{4}{*}{4.0} & 0 & 1095 & 62 & 960 & 30 \\
\hline & & 7 & 1978 & 21 & 1125 & 11 \\
\hline & & 14 & 393 & 26 & 393 & 10 \\
\hline & & 30 & 175 & 11 & 168 & 9 \\
\hline & \multirow[t]{4}{*}{10} & 0 & 461 & 2 & 461 & 2 \\
\hline & & 7 & $\mathrm{nt}$ & $\mathrm{nt}$ & 91 & 7 \\
\hline & & 14 & $\mathrm{nt}$ & $\mathrm{nt}$ & 185 & 6 \\
\hline & & 30 & 478 & 7 & $\mathrm{nt}$ & $\mathrm{nt}$ \\
\hline
\end{tabular}

Assessment of particulate content using light obscuration techniques in two PF-SZ-IFX batches reconstituted to $10 \mathrm{mg} / \mathrm{mL}$ and subsequently diluted in polyethylene saline solution infusion bags to 0.4 and $4.0 \mathrm{mg} / \mathrm{mL}$. Samples were stored at $5 \pm 3{ }^{\circ} \mathrm{C}$ or $25 \pm 2{ }^{\circ} \mathrm{C}(60 \% \pm 5 \% \mathrm{RH})$ for 7,14 or 30 days

$n t$ not tested, $P F-S Z$-IFX PF-06438179/GP1111, $R H$ relative humidity
$C=\frac{A_{\text {corr }} \times \mathrm{DF}}{\varepsilon_{\text {PF-SZ-IFX }} \times 1}$

where $C=$ protein concentration $(\mathrm{mg} / \mathrm{mL}), \mathrm{DF}=$ dilution factor, $\varepsilon_{\mathrm{PF}-\mathrm{SZ}-\mathrm{IFX}}=\mathrm{PF}$-SZ-IFX-specific extinction coefficient $\left[(\mathrm{mg} / \mathrm{mL})^{-1} \mathrm{~cm}^{-1}\right]$ and $\mathrm{l}=$ path length .

\subsection{Charge Heterogeneity}

Charge heterogeneity was evaluated because charge variants representing protein modifications can potentially affect protein function and biological activity. Charge heterogeneity assessment was performed by imaged capillary isoelectric focusing (iCE) using a ProteinSimple iCE3 ${ }^{\mathrm{TM}}$ analyser (ProteinSimple, USA) equipped with a PrinCE autosampler. Reconstituted samples were diluted with water to a final concentration of $2 \mathrm{mg} / \mathrm{mL}$ before measurement. Due to interference with $\mathrm{NaCl}$ solution, desalting was performed by ultrafiltration using Amicon $30 \mathrm{kDa}$ filter units (Millipore, Merck, Germany) for desalting and ultrapure water as diluent. After centrifugation at $1000 \mathrm{rcf}(\mathrm{G})$ for $10 \mathrm{~min}$, desalting was achieved, and protein was separated from matrix before measurement for 0.4 and $4.0 \mathrm{mg} / \mathrm{mL}$ concentrations.

Solution $(30 \mu \mathrm{L})$ was denatured by dilution into $170 \mu \mathrm{L}$ of iCE injection diluent (50 $\mu \mathrm{L} \mathrm{1 \%} \mathrm{methyl} \mathrm{cellulose,} 8 \mu \mathrm{L}$ Pharmalyte [isoelectric point $\{\mathrm{pH}(\mathrm{I})\}: 3-10], 1 \mu \mathrm{L} \mathrm{pH}(\mathrm{I})$ marker $[\mathrm{pH}(\mathrm{I})=6.14], 1 \mu \mathrm{L} \mathrm{pH}(\mathrm{I})$ marker $[\mathrm{pH}(\mathrm{I})=9.50], 50 \mu \mathrm{L}$ $8 \mathrm{M}$ urea, $60 \mu \mathrm{L}$ water). Sample mixture was injected onto a film-coated iCE cartridge ( $100 \mu \mathrm{M}$ inner diameter $\times 50 \mathrm{~mm}$; ProteinSimple) and focused by applying a $1500 \mathrm{~V}$ potential for $1 \mathrm{~min}$ (pre-focusing step) followed by $3000 \mathrm{~V}$ for $6 \mathrm{~min}$ (focusing step). Absorbance was monitored at $280 \mathrm{~nm}$. 
Table 3 Ultraviolet spectrophotometric analysis of protein concentration for PF-SZ-IFX batches following in-use preparation for intravenous infusion

\begin{tabular}{|c|c|c|c|c|}
\hline Batch & $\begin{array}{l}\text { Theoretical infliximab } \\
\text { conc. }(\mathrm{mg} / \mathrm{mL})\end{array}$ & Time (days) & $\begin{array}{l}5{ }^{\circ} \mathrm{C} \\
\text { Measured infliximab } \\
\text { conc. }(\mathrm{mg} / \mathrm{mL})\end{array}$ & $\begin{array}{l}25^{\circ} \mathrm{C} / 60 \% \mathrm{RH} \\
\text { Measured inflixi- } \\
\text { mab conc. (mg/ } \\
\mathrm{mL})\end{array}$ \\
\hline \multirow[t]{15}{*}{ A } & 0.4 & 0 & 0.38 & 0.38 \\
\hline & & 7 & 0.36 & 0.36 \\
\hline & & 14 & 0.37 & 0.37 \\
\hline & & 30 & 0.37 & 0.37 \\
\hline & & RSD & $2.2 \%$ & $2.2 \%$ \\
\hline & 4 & 0 & 3.86 & 3.84 \\
\hline & & 7 & 3.85 & 3.84 \\
\hline & & 14 & 3.85 & 3.84 \\
\hline & & 30 & 3.86 & 3.84 \\
\hline & & RSD & $0.1 \%$ & $0.0 \%$ \\
\hline & 10 & 0 & 10.03 & 10.03 \\
\hline & & 7 & $\mathrm{nt}$ & 9.77 \\
\hline & & 14 & $\mathrm{nt}$ & 9.78 \\
\hline & & 30 & 9.67 & $\mathrm{nt}$ \\
\hline & & RSD & $2.6 \%$ & $1.5 \%$ \\
\hline \multirow[t]{15}{*}{ B } & 0.4 & 0 & 0.38 & 0.39 \\
\hline & & 7 & 0.37 & 0.36 \\
\hline & & 14 & 0.37 & 0.37 \\
\hline & & 30 & 0.36 & 0.37 \\
\hline & & RSD & $2.2 \%$ & $3.4 \%$ \\
\hline & 4 & 0 & 3.84 & 3.85 \\
\hline & & 7 & 3.84 & 3.85 \\
\hline & & 14 & 3.83 & 3.85 \\
\hline & & 30 & 3.84 & 3.85 \\
\hline & & RSD & $0.1 \%$ & $0.0 \%$ \\
\hline & 10 & 0 & 9.96 & 9.96 \\
\hline & & 7 & $\mathrm{nt}$ & 9.95 \\
\hline & & 14 & $\mathrm{nt}$ & 9.86 \\
\hline & & 30 & 9.87 & $\mathrm{nt}$ \\
\hline & & RSD & $0.6 \%$ & $0.6 \%$ \\
\hline
\end{tabular}

Assessment of protein concentration using UV spectrometry in two PF-SZ-IFX batches reconstituted to $10 \mathrm{mg} / \mathrm{mL}$ and subsequently diluted in polyethylene saline solution infusion bags to 0.4 and $4.0 \mathrm{mg} / \mathrm{mL}$. Samples were stored at $5 \pm 3{ }^{\circ} \mathrm{C}$ or $25 \pm 2{ }^{\circ} \mathrm{C}(60 \% \pm 5 \% \mathrm{RH})$ for 7,14 or 30 days. Pre-defined acceptance criteria for 0.4 and $4.0 \mathrm{mg} / \mathrm{mL}$ samples: $\pm 10 \%$ of T0 concentration

$n t$ not tested, $P F-S Z-I F X$ PF-06438179/GP1111, $R H$ relative humidity, $R S D$ relative standard deviation, $U V$ ultraviolet

\subsection{Purity}

Size exclusion chromatography (SEC) and reducing capillary electrophoresis sodium dodecyl sulphate (CE-SDS) were used to evaluate molecular weight variants, which are sensitive to changes in storage conditions and can potentially affect the immunogenicity and potency of medicines. SEC was used to assess the formation of high-molecular-weight variants; experiments were conducted as reported previously [7]. Samples with a concentration of $0.4 \mathrm{mg} / \mathrm{mL}$ were measured undiluted; samples with a concentration of 4.0 or
$10 \mathrm{mg} / \mathrm{mL}$ were diluted with mobile phase to a concentration of $1.0 \mathrm{mg} / \mathrm{mL}$ before measurement.

CE-SDS was employed as a canonical method because of its ability to more completely resolve incomplete antibody fragments and low-molecular-weight variants compared with SEC. Analysis was performed on a PA800 Plus (Beckman Coulter) equipped with a photodiode array detector. Samples were diluted to $1 \mathrm{mg} / \mathrm{mL}$ using sample buffer containing SDS and 2-mercaptoethanol; desalting was performed for 0.4 and $4.0 \mathrm{mg} / \mathrm{mL}$ concentration samples. The mixture was heated at $65^{\circ} \mathrm{C}$ for $10 \mathrm{~min}$. Prior to separation, a bare fused 
Table 4 Imaged capillary isoelectric focusing analysis of PF-SZ-IFX batches following in-use preparation for intravenous infusion

\begin{tabular}{|c|c|c|c|c|c|c|c|c|}
\hline \multirow[t]{2}{*}{ Batch } & \multirow{2}{*}{$\begin{array}{l}\text { Theoretical inflixi- } \\
\text { mab, conc. }(\mathrm{mg} / \mathrm{mL})\end{array}$} & \multirow[t]{2}{*}{ Time (days) } & \multicolumn{3}{|l|}{$5^{\circ} \mathrm{C}$} & \multicolumn{3}{|l|}{$25^{\circ} \mathrm{C} / 60 \% \mathrm{RH}$} \\
\hline & & & Main species & Acidic species & Basic species & Main species & Acidic species & Basic species \\
\hline \multirow[t]{15}{*}{ A } & \multirow[t]{5}{*}{0.4} & 0 & $\mathrm{na}^{\mathrm{a}}$ & & & & & \\
\hline & & 7 & 61 & 18 & 21 & 60 & 18 & 21 \\
\hline & & 14 & 60 & 19 & 21 & 59 & 20 & 21 \\
\hline & & 30 & 60 & 19 & 21 & 59 & 21 & 21 \\
\hline & & RSD & 1.0 & 3.1 & 0.0 & 1.0 & 7.8 & 0.0 \\
\hline & \multirow[t]{5}{*}{4} & 0 & $\mathrm{na}^{\mathrm{a}}$ & & & & & \\
\hline & & 7 & 61 & 18 & 21 & 59 & 20 & 21 \\
\hline & & 14 & 60 & 19 & 22 & 59 & 20 & 21 \\
\hline & & 30 & 60 & 19 & 21 & 59 & 21 & 21 \\
\hline & & RSD & 1.0 & 3.1 & 2.7 & 0.0 & 2.8 & 0.0 \\
\hline & \multirow[t]{5}{*}{10} & 0 & 55 & 24 & 21 & 55 & 24 & 21 \\
\hline & & 7 & $\mathrm{nt}$ & $\mathrm{nt}$ & nt & 49 & 32 & 19 \\
\hline & & 14 & $\mathrm{nt}$ & $\mathrm{nt}$ & $\mathrm{nt}$ & 49 & 32 & 19 \\
\hline & & 30 & 50 & 31 & 19 & nt & nt & $\mathrm{nt}$ \\
\hline & & RSD & 6.7 & 18.0 & 7.1 & 6.8 & 15.7 & 5.9 \\
\hline \multirow[t]{15}{*}{ B } & \multirow[t]{5}{*}{0.4} & 0 & $\mathrm{na}^{\mathrm{a}}$ & & & & & \\
\hline & & 7 & 60 & 19 & 21 & 60 & 19 & 21 \\
\hline & & 14 & 61 & 18 & 21 & 59 & 20 & 21 \\
\hline & & 30 & 60 & 18 & 21 & 58 & 21 & 21 \\
\hline & & RSD & 1.0 & 3.1 & 0.0 & 1.7 & 5.0 & 0.0 \\
\hline & \multirow[t]{5}{*}{4} & 0 & $\mathrm{na}^{\mathrm{a}}$ & & & & & \\
\hline & & 7 & 61 & 19 & 21 & 60 & 19 & 21 \\
\hline & & 14 & 59 & 19 & 21 & 59 & 20 & 21 \\
\hline & & 30 & 60 & 19 & 21 & 58 & 21 & 21 \\
\hline & & RSD & 1.7 & 0.0 & 0.0 & 1.7 & 5.0 & 0.0 \\
\hline & \multirow[t]{5}{*}{10} & 0 & 54 & 25 & 21 & 54 & 25 & 21 \\
\hline & & 7 & $\mathrm{nt}$ & $\mathrm{nt}$ & $\mathrm{nt}$ & 49 & 32 & 19 \\
\hline & & 14 & $\mathrm{nt}$ & $\mathrm{nt}$ & $\mathrm{nt}$ & 48 & 33 & 19 \\
\hline & & 30 & 49 & 32 & 19 & $\mathrm{nt}$ & $\mathrm{nt}$ & $\mathrm{nt}$ \\
\hline & & RSD & 6.9 & 17.4 & 7.1 & 6.4 & 14.5 & 5.9 \\
\hline
\end{tabular}

Data are presented as \% unless otherwise indicated

Assessment of charge heterogeneity using iCE in two PF-SZ-IFX batches reconstituted to $10 \mathrm{mg} / \mathrm{mL}$ and subsequently diluted in polyethylene saline solution infusion bags to 0.4 and $4.0 \mathrm{mg} / \mathrm{mL}$. Samples were stored at $5 \pm 3{ }^{\circ} \mathrm{C}$ or $25 \pm 2{ }^{\circ} \mathrm{C}(60 \% \pm 5 \% \mathrm{RH})$ for 7,14 , or 30 days. The relative abundance of main, acidic and basic species are given. to data were taken from lot release data, samples were not diluted or desalted before analysis. t0 data were not used as comparator for diluted samples due to differences in sample preparation

$i C E$ imaged capillary isoelectric focusing, $n a$ not applicable, $n t$ not tested, $P F-S Z-I F X$ PF-06438179/GP1111, $R H$ relative humidity, $R S D$ relative standard deviation

${ }^{a}$ Values at day 0 were not determined for diluted samples because only reconstituted material was used at this timepoint (release data)

silica capillary (internal diameter $50 \mu \mathrm{M}$ ) was rinsed with $0.1 \mathrm{~mol} / \mathrm{L} \mathrm{NaOH}$ for $3 \mathrm{~min}$ and $0.1 / \mathrm{L} \mathrm{HCl}$ for $1 \mathrm{~min}$, followed by water in the reverse direction. The capillary was filled with SDS gel buffer for $10 \mathrm{~min}$ (reverse direction), and samples were injected at $5 \mathrm{kV}$ for $50 \mathrm{~s}$ and separated at $15 \mathrm{kV}$ for $30 \mathrm{~min}$.

\subsection{Potency}

The functional activity of PF-SZ-IFX was determined using a validated cell-based assay, as reported previously [7], to verify that samples remained functionally stable under extended in-use conditions. 
Fig. 1 Representative iCE overlay of PF-SZ-IFX samples reconstituted to $10 \mathrm{mg} / \mathrm{mL}$ and then diluted to (a) $0.4 \mathrm{mg} / \mathrm{mL}$ and (b) $4.0 \mathrm{mg} / \mathrm{mL}$ in polyethylene saline solution infusion bags. All samples were from batch $\mathrm{A}$ and stored at 5 or $25^{\circ} \mathrm{C}$ for up to 30 days. $i C E$ imaged capillary isoelectric focusing, $p H(I)$ isoelectric point, $P F-S Z$ IFX PF-06438179/GP1111

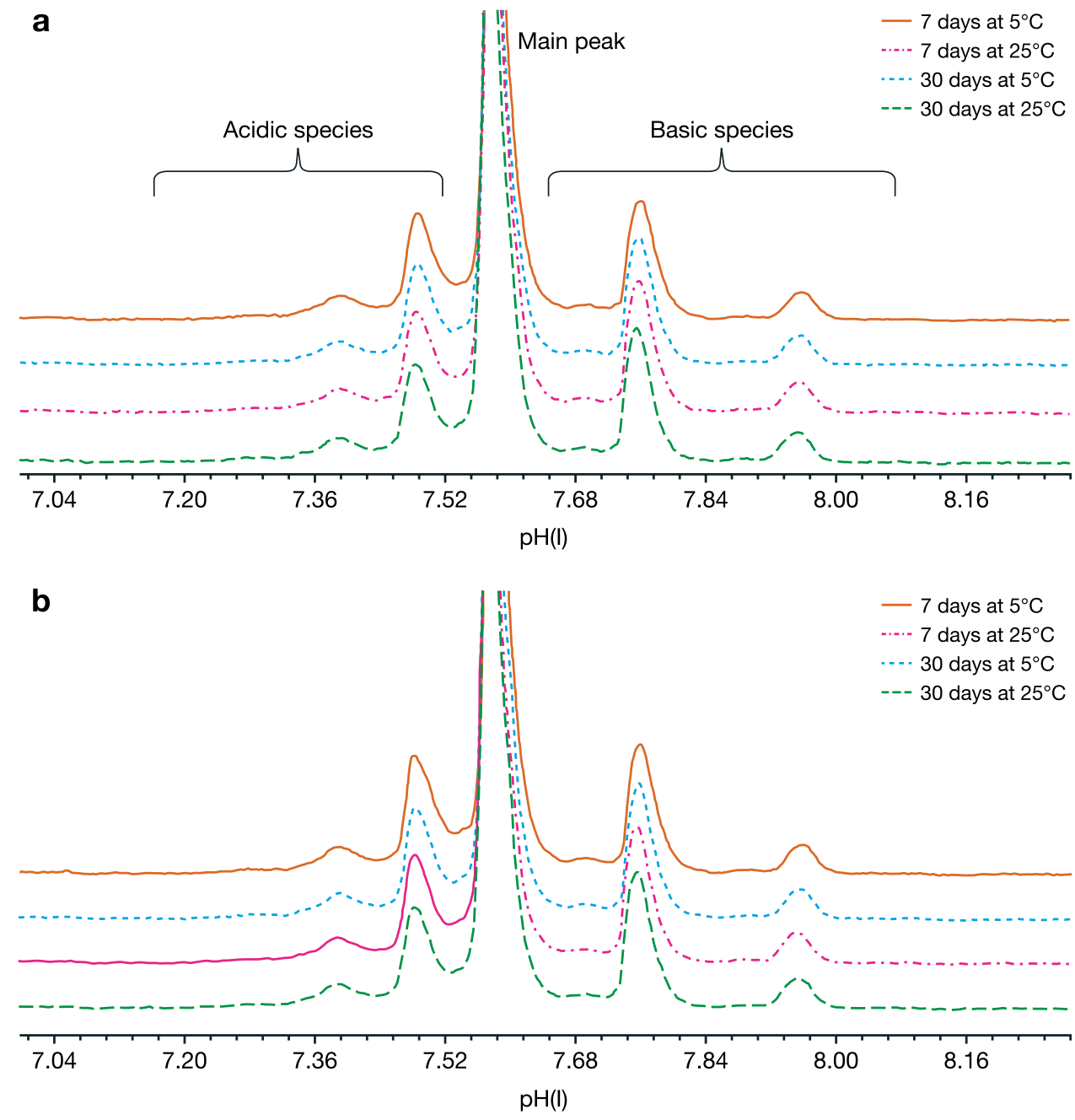

\subsection{Acceptance Criteria, Method Validation and Data Analysis}

Acceptance criteria were defined according to methodological guidelines issued by the International Conference on Harmonization (ICH guidelines for stability testing [13] and specifications for biotechnological/biological products [22]), Eur Ph [18] and European Medicines Agencyapproved commercial release and shelf-life specifications for PF-SZ-IFX.

Analytical methods used in the study were either verified (compendial methods) or fully validated according to ICH guidelines on validation of analytical procedures [23], Eur $\mathrm{Ph}$ [18] and USP guidelines [21]. Specificity, accuracy, precision and detection limits of all methods were determined using multiple samples during method validation in accordance with ICH guidelines on validation of analytical procedures [23]. For the potency test, duplicates were used as per routine procedure in the quality control laboratory. Potency was determined using the worst-case approach (by testing the samples at the end of storage).

\section{Results}

\subsection{Osmolality, pH and Appearance}

Table 1 presents the osmolality, $\mathrm{pH}$ and clarity across batches, reconstituted/diluted solutions and storage conditions/durations; all results met pre-defined acceptance criteria, and no changes were observed upon dilution and storage. All samples were colourless to brownish yellow in appearance and met the acceptance criteria of not more intense than reference standard B5 [18].

\subsection{Sub-Visible Particles}

Analysis of the solutions by light obscuration identified no changes in sub-visible particle content across batches, reconstituted/diluted solutions and storage conditions/durations (Table 2). The 0.4 and $4.0 \mathrm{mg} / \mathrm{mL}$ samples contained $\leq 2105$ particles of $\leq 10 \mu \mathrm{m}$ and $\leq 433$ particles of $\leq 25 \mu \mathrm{m}$. The $10 \mathrm{mg} / \mathrm{mL}$ samples contained $\leq 638$ particles of $\leq 10 \mu \mathrm{m}$ 
Table 5 Size exclusion chromatography analysis of PF-SZ-IFX batches following in-use preparation for intravenous infusion

\begin{tabular}{|c|c|c|c|c|c|c|}
\hline \multirow[t]{2}{*}{ Batch } & \multirow{2}{*}{$\begin{array}{l}\text { Theoretical infliximab, } \\
\text { conc. }(\mathrm{mg} / \mathrm{mL})\end{array}$} & \multirow[t]{2}{*}{ Time (days) } & \multicolumn{2}{|l|}{$5^{\circ} \mathrm{C}$} & \multicolumn{2}{|c|}{$25^{\circ} \mathrm{C} / 60 \% \mathrm{RH}$} \\
\hline & & & Monomer & HMMS & Monomer & HMMS \\
\hline \multirow[t]{12}{*}{ A } & \multirow[t]{4}{*}{0.4} & 0 & 99.7 & $<0.3$ & 99.7 & $<0.3$ \\
\hline & & 7 & 99.7 & $<0.3$ & 99.8 & $<0.3$ \\
\hline & & 14 & 99.8 & $<0.3$ & 99.8 & $<0.3$ \\
\hline & & 30 & 99.8 & $<0.3$ & 99.7 & $<0.3$ \\
\hline & \multirow[t]{4}{*}{4.0} & 0 & 99.7 & $<0.3$ & 99.7 & $<0.3$ \\
\hline & & 7 & 99.8 & $<0.3$ & 99.8 & $<0.3$ \\
\hline & & 14 & 99.8 & $<0.3$ & 99.8 & $<0.3$ \\
\hline & & 30 & 99.8 & $<0.3$ & 99.8 & $<0.3$ \\
\hline & \multirow[t]{4}{*}{10} & 0 & 99.7 & $<0.3$ & 99.7 & $<0.3$ \\
\hline & & 7 & $\mathrm{nt}$ & nt & 99.8 & $<0.3$ \\
\hline & & 14 & $\mathrm{nt}$ & $\mathrm{nt}$ & 99.8 & $<0.3$ \\
\hline & & 30 & 99.8 & $<0.3$ & $\mathrm{nt}$ & $\mathrm{nt}$ \\
\hline \multirow[t]{12}{*}{ B } & \multirow[t]{4}{*}{0.4} & 0 & 99.7 & $<0.3$ & 99.7 & $<0.3$ \\
\hline & & 7 & 99.8 & $<0.3$ & 99.8 & $<0.3$ \\
\hline & & 14 & 99.8 & $<0.3$ & 99.8 & $<0.3$ \\
\hline & & 30 & 99.8 & $<0.3$ & 99.7 & $<0.3$ \\
\hline & \multirow[t]{4}{*}{4.0} & 0 & 99.7 & $<0.3$ & 99.7 & $<0.3$ \\
\hline & & 7 & 99.9 & $<0.3$ & 99.8 & $<0.3$ \\
\hline & & 14 & 99.8 & $<0.3$ & 99.8 & $<0.3$ \\
\hline & & 30 & 99.8 & $<0.3$ & 99.8 & $<0.3$ \\
\hline & \multirow[t]{4}{*}{10} & 0 & 99.7 & $<0.3$ & 99.7 & $<0.3$ \\
\hline & & 7 & $\mathrm{nt}$ & nt & 99.8 & $<0.3$ \\
\hline & & 14 & $\mathrm{nt}$ & $\mathrm{nt}$ & 99.8 & $<0.3$ \\
\hline & & 30 & 99.8 & $<0.3$ & $\mathrm{nt}$ & $\mathrm{nt}$ \\
\hline
\end{tabular}

Data are presented as \% unless otherwise indicated

Assessment of molecular weight variants using SEC in two PF-SZ-IFX batches reconstituted to $10 \mathrm{mg} /$ $\mathrm{mL}$ and subsequently diluted in saline solution infusion bags to 0.4 and $4.0 \mathrm{mg} / \mathrm{mL}$. Samples were stored at $5 \pm 3{ }^{\circ} \mathrm{C}$ or $25 \pm 2{ }^{\circ} \mathrm{C}(60 \pm 5 \% \mathrm{RH})$ for 7,14 or 30 days. The relative abundance of HMMS, and purity (monomer) are given

$H M M S$ high molecular weight variant, $n t$ not tested, $P F-S Z-I F X$ PF-06438179/GP1111, $R H$ relative humidity, $S E C$ size exclusion chromatography and $\leq 9$ particles of $\leq 25 \mu \mathrm{m}$. All results met compendia requirements [21].

\subsection{Protein Concentration by Ultraviolet}

At each sample concentration, no notable changes in protein concentration were observed over time across batches and storage conditions tested (Table 3). Absence of changes in UV absorption indicated minimal protein aggregation, precipitation and adsorption under in-use conditions. All results met pre-defined acceptance criteria.

\subsection{Charge Heterogeneity by Imaged Capillary Isoelectric Focusing}

No significant differences in charge variants between individual timepoints were identified for diluted samples (i.e. 0.4 and $4.0 \mathrm{mg} / \mathrm{mL}$ ) for the 5 or $25{ }^{\circ} \mathrm{C} / 60 \% \mathrm{RH}$ conditions; the pre-defined acceptance criteria were met for all measurements (Table 4). Further comparison of the electropherograms revealed no observable differences in acidic and basic species upon storage of diluted samples (Fig. 1). A slight decrease in main species and increase of acidic species (within pre-defined limits) occurred with time for reconstituted solutions $(10 \mathrm{mg} / \mathrm{mL})$ stored at $5{ }^{\circ} \mathrm{C}$ for 30 days and at $25{ }^{\circ} \mathrm{C} / 60 \% \mathrm{RH}$ for 14 days (Table 4), with no changes in basic species. The acidic species were characterised during development and found to comprise PF-SZ-IFX with charged glycans and elevated glycation and deamidation, 
Fig. 2 Representative size exclusion chromatography overlay of PF-SZ-IFX samples reconstituted to $10 \mathrm{mg} / \mathrm{mL}$ and diluted to a $0.4 \mathrm{mg} / \mathrm{mL}$ and b $4.0 \mathrm{mg} / \mathrm{mL}$ in polyethylene saline solution infusion bags. All samples were from batch A and stored at 5 or $25^{\circ} \mathrm{C}$ for 7 , 14 (data not shown) or 30 days. HMMS high-molecular-weight variant, $L M M S$ low-molecularweight variant, $P F-S Z-I F X$ PF-06438179/GP1111

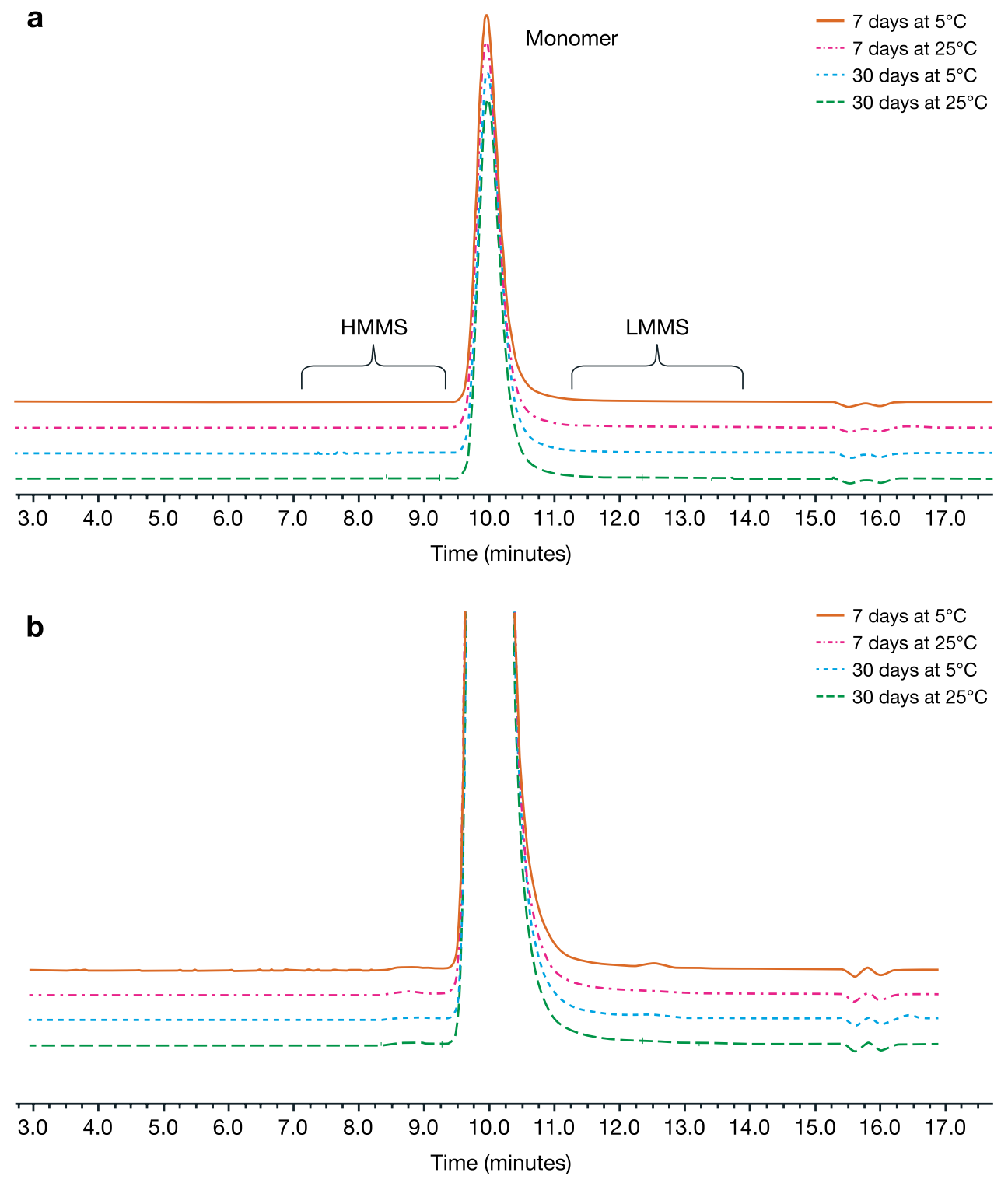

whereas basic species were found to comprise PF-SZ-IFX mono- and di-C-terminal lysine species [7].

\subsection{Purity by Size Exclusion Chromatography and Reducing Capillary Electrophoresis Sodium Dodecyl Sulphate}

SEC analysis revealed no changes in high-molecular-weight variants (Table 5), for which the mass was indicative of a dimer, consistent with previous observations [7]. Further comparison of the chromatograms revealed no observable differences in a high-molecular-weight species upon storage of diluted samples (Fig. 2). Across batches and storage conditions/durations, the monomer content of all samples was $\geq 99.7 \%$, and the high-molecular-weight variant content (i.e. aggregates) of all samples was below the limit of quantification for the method $(<0.3 \%)$; all results were within the pre-defined acceptance criteria. No low-molecular-weight species were detected by SEC.

CE-SDS analysis showed that the abundance of fragments was low $(0.4$ and $4.0 \mathrm{mg} / \mathrm{mL}$ samples: $\leq 2.1 \% ; 10 \mathrm{mg} /$ $\mathrm{mL}$ samples: $\leq 1.7 \%)$ and purity was high $(0.4$ and $4.0 \mathrm{mg} /$ mL samples: $\geq 97.9 \% ; 10 \mathrm{mg} / \mathrm{mL}$ samples: $\geq 98.0 \%$ ) across batches and storage conditions/durations tested (Table 6; Fig. 3). All results met pre-defined acceptance criteria.

\subsection{Potency}

Analysis of bioassay data for reconstituted/diluted samples showed no changes in potency across batches and storage 
Table 6 Reducing CE-SDS analysis of PF-SZ-IFX batches following in-use preparation for intravenous infusion

\begin{tabular}{|c|c|c|c|c|c|c|}
\hline \multirow[t]{2}{*}{ Batch } & \multirow{2}{*}{$\begin{array}{l}\text { Theoretical inflixi- } \\
\text { mab, conc. }(\mathrm{mg} / \mathrm{mL})\end{array}$} & \multirow[t]{2}{*}{ Time (days) } & \multicolumn{2}{|l|}{$5{ }^{\circ} \mathrm{C}$} & \multicolumn{2}{|c|}{$25^{\circ} \mathrm{C} / 60 \% \mathrm{RH}$} \\
\hline & & & Main peak & $\overline{\text { Fragments }}$ & Main peak & Fragments \\
\hline \multirow[t]{15}{*}{ A } & \multirow[t]{5}{*}{0.4} & 0 & 98.6 & 1.4 & 98.6 & 1.4 \\
\hline & & 7 & 98.6 & 1.4 & 98.5 & 1.5 \\
\hline & & 14 & 98.3 & 1.7 & 98.4 & 1.6 \\
\hline & & 30 & 98.2 & 1.8 & 98.1 & 1.9 \\
\hline & & RSD & 0.2 & 13.1 & 0.2 & 13.5 \\
\hline & \multirow[t]{5}{*}{4} & 0 & 98.6 & 1.4 & 98.6 & 1.4 \\
\hline & & 7 & 98.4 & 1.6 & 98.4 & 1.6 \\
\hline & & 14 & 98.3 & 1.7 & 97.9 & 2.1 \\
\hline & & 30 & 98.0 & 1.7 & 98.2 & 1.8 \\
\hline & & RSD & 0.3 & 8.8 & 0.3 & 17.3 \\
\hline & \multirow[t]{5}{*}{10} & 0 & 98.6 & 1.4 & 98.6 & 1.4 \\
\hline & & 7 & $\mathrm{nt}$ & $\mathrm{nt}$ & 98.2 & 1.6 \\
\hline & & 14 & $\mathrm{nt}$ & nt & 98.4 & 1.6 \\
\hline & & 30 & 98.0 & 1.6 & $\mathrm{nt}$ & $\mathrm{nt}$ \\
\hline & & RSD & 0.4 & 9.4 & 0.2 & 7.5 \\
\hline \multirow[t]{15}{*}{ B } & \multirow[t]{5}{*}{0.4} & 0 & 98.6 & 1.4 & 98.6 & 1.4 \\
\hline & & 7 & na & na & 98.3 & 1.7 \\
\hline & & 14 & 98.4 & 1.6 & 98.0 & 1.7 \\
\hline & & 30 & 98.4 & 1.6 & 98.4 & 1.6 \\
\hline & & RSD & 0.1 & 7.5 & 0.3 & 8.8 \\
\hline & \multirow[t]{5}{*}{4} & 0 & 98.6 & 1.4 & 98.6 & 1.4 \\
\hline & & 7 & 98.3 & 1.7 & 98.2 & 1.8 \\
\hline & & 14 & 98.1 & 1.9 & 98.1 & 1.9 \\
\hline & & 30 & 98.3 & 1.7 & 98.0 & 2.0 \\
\hline & & RSD & 0.2 & 12.3 & 0.3 & 14.8 \\
\hline & \multirow[t]{5}{*}{10} & 0 & 98.6 & 1.4 & 98.6 & 1.4 \\
\hline & & 7 & $\mathrm{nt}$ & $\mathrm{nt}$ & 98.4 & 1.6 \\
\hline & & 14 & $\mathrm{nt}$ & $\mathrm{nt}$ & 98.3 & 1.7 \\
\hline & & 30 & 98.4 & 1.6 & $\mathrm{nt}$ & $\mathrm{nt}$ \\
\hline & & RSD & 0.1 & 9.4 & 0.2 & 9.8 \\
\hline
\end{tabular}

Data are presented as \% unless otherwise indicated

Assessment of molecular weight variants using reducing CE-SDS in two PF-SZ-IFX batches reconstituted to $10 \mathrm{mg} / \mathrm{mL}$ and subsequently diluted in polyethylene saline solution infusion bags to 0.4 and $4.0 \mathrm{mg} / \mathrm{mL}$. Samples were stored at $5 \pm 3{ }^{\circ} \mathrm{C}$ or $25 \pm 2{ }^{\circ} \mathrm{C}(60 \% \pm 5 \% \mathrm{RH})$ for 7,14 or 30 days. The relative abundance of fragments and the purity (HC plus LC) are given

$C E-S D S$ sodium dodecyl sulphate capillary electrophoresis, $H C$ heavy chain, $L C$ light chain, $n a$ results not applicable, $n t$ not tested, $P F-S Z-I F X$ PF-06438179/GP1111, $R H$ relative humidity, $R S D$ relative standard deviation conditions/durations (Table 7). All results were within assay variability limits and met pre-defined acceptance criteria.

\section{Discussion}

General in-use stability data are evaluated in the assessment process before biosimilars are approved, but extended in-use handling (e.g. longer storage of the diluted preparations in infusion bags), which depends upon the conditions of preparation, is not covered. Therefore, we evaluated the extended in-use stability of the infliximab biosimilar PFSZ-IFX. State-of-the-art analytical techniques were used to assess biological and physicochemical stability following reconstitution, dilution in polyethylene saline infusion bags and subsequent storage of reconstituted and diluted samples, simulating worst-case conditions.

Osmolality, $\mathrm{pH}$, clarity and particulate content (visible and sub-visible) met pre-defined acceptability criteria under all conditions tested for up to 30 days. Assessment of samples at $0,7,14$ and 30 days by UV spectrometry revealed no change in protein concentration, demonstrating that 
Fig. 3 Representative capillary electrophoresis sodium dodecyl sulphate overlay of PF-SZIFX samples reconstituted to $10 \mathrm{mg} / \mathrm{mL}$ and diluted to $\mathbf{a}$ $0.4 \mathrm{mg} / \mathrm{mL}$ and $\mathbf{b} 4.0 \mathrm{mg} / \mathrm{mL}$ in polyethylene saline solution infusion bags. All samples were from batch A and stored at 5 or $25^{\circ} \mathrm{C}$ for 7,14 (data not shown) or 30 days. PF-SZ-IFX PF-06438179/GP1111
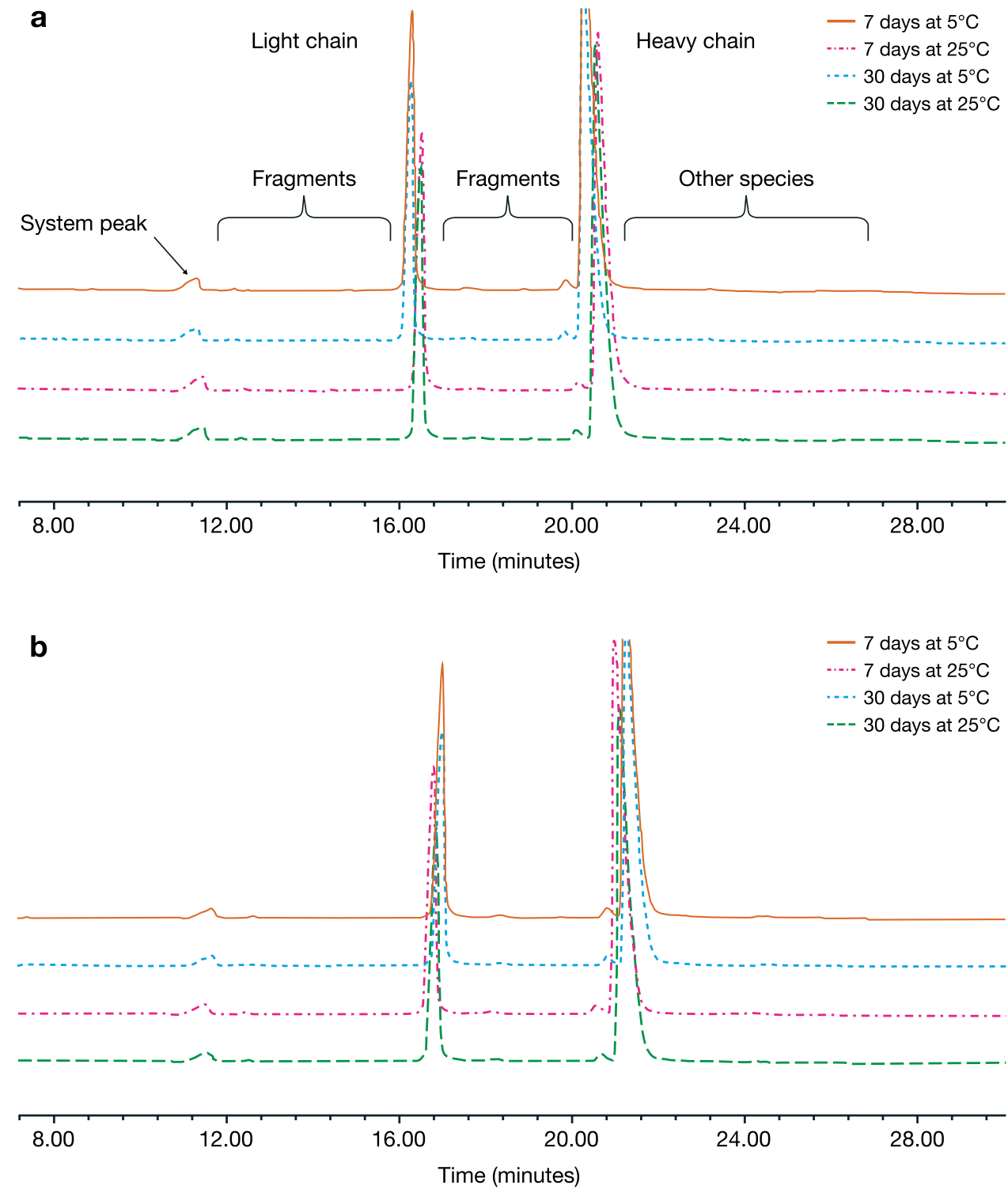

no precipitation or measurable adsorption of PF-SZ-IFX occurred under in-use conditions for intravenous infusion. A lack of adsorption is particularly important because protein adsorption to solid surfaces can compromise intended soluble doses, especially at low concentrations [24].

Charge variants represent protein modifications ranging from inert (e.g. C-terminal lysine residues [basic species]) to those affecting protein function and biological activity (e.g. deamidations at critical asparagine residues [acidic species]) $[25,26]$. In the present study, using iCE, no effect of in-use dilution on charge variants was observed. A slight decrease in main species and increase of acidic species occurred over time with solutions reconstituted to $10 \mathrm{mg} / \mathrm{mL}$ and stored at $25{ }^{\circ} \mathrm{C} / 60 \% \mathrm{RH}$ for 14 days or at $5^{\circ} \mathrm{C}$ for 30 days. This represents a worst-case scenario unlikely to be encountered in clinical practice because PF-SZ-IFX is intended for immediate use and should be further diluted within the infusion concentration range upon reconstitution (and not stored as reconstituted solution). No changes were observed in the lower concentration diluted samples $(0.4$ or $4.0 \mathrm{mg} / \mathrm{mL})$ for up to 30 days of storage at both 5 and $25{ }^{\circ} \mathrm{C} / 60 \% \mathrm{RH}$. Characterisation of the PF-SZ-IFX charge variants were consistent with those reported for reference infliximab [27]. In forced degradation studies, where reconstituted PF-SZIFX was incubated at $40{ }^{\circ} \mathrm{C}$ (data not shown), we identified deamidation as the main degradation pathway potentially impacting the acidic species; these findings are consistent with previous observations [27-29]. Several asparagine (Asn) residues are located at the interface between TNF- $\alpha$ and infliximab, particularly HC Asn57 in HC CDR2 [30], and a potential correlation between elevated deamidation and a loss of potency has previously been demonstrated [29]. Importantly, when we evaluated potency using a validated, in-house bioassay, samples remained functionally stable 
Table 7 Potency of PF-SZ-IFX batches following in-use preparation for intravenous infusion

\begin{tabular}{|c|c|c|c|c|}
\hline \multirow[t]{2}{*}{ Batch } & \multirow{2}{*}{$\begin{array}{l}\text { Theoretical inflixi- } \\
\text { mab conc. }(\mathrm{mg} / \mathrm{mL})\end{array}$} & \multirow[t]{2}{*}{ Time (days) } & \multicolumn{2}{|c|}{ Potency (\%) } \\
\hline & & & $5{ }^{\circ} \mathrm{C}$ & $25^{\circ} \mathrm{C} / 60 \% \mathrm{RH}$ \\
\hline \multirow[t]{12}{*}{ A } & \multirow[t]{4}{*}{0.4} & 0 & 103 & 103 \\
\hline & & 7 & $\mathrm{nt}$ & nt \\
\hline & & 14 & nt & nt \\
\hline & & 30 & 96 & 93 \\
\hline & \multirow[t]{4}{*}{4.0} & 0 & 103 & 103 \\
\hline & & 7 & nt & $\mathrm{nt}$ \\
\hline & & 14 & nt & $\mathrm{nt}$ \\
\hline & & 30 & 103 & 102 \\
\hline & \multirow[t]{4}{*}{10} & 0 & 103 & 103 \\
\hline & & 7 & nt & $\mathrm{nt}$ \\
\hline & & 14 & nt & 104 \\
\hline & & 30 & 105 & $\mathrm{nt}$ \\
\hline \multirow[t]{12}{*}{ B } & \multirow[t]{4}{*}{0.4} & 0 & 102 & 102 \\
\hline & & 7 & $\mathrm{nt}$ & $\mathrm{nt}$ \\
\hline & & 14 & nt & $\mathrm{nt}$ \\
\hline & & 30 & 95 & 93 \\
\hline & \multirow[t]{4}{*}{4.0} & 0 & 102 & 102 \\
\hline & & 7 & $\mathrm{nt}$ & $\mathrm{nt}$ \\
\hline & & 14 & nt & $\mathrm{nt}$ \\
\hline & & 30 & 99 & 101 \\
\hline & \multirow[t]{4}{*}{10} & 0 & 102 & 102 \\
\hline & & 7 & $\mathrm{nt}$ & $\mathrm{nt}$ \\
\hline & & 14 & nt & 109 \\
\hline & & 30 & 110 & $\mathrm{nt}$ \\
\hline
\end{tabular}

Assessment of potency by bioassay in two PF-SZ-IFX batches reconstituted to $10 \mathrm{mg} / \mathrm{mL}$ and subsequently diluted in polyethylene saline solution infusion bags to 0.4 and $4.0 \mathrm{mg} / \mathrm{mL}$. Samples were stored at $5 \pm 3{ }^{\circ} \mathrm{C}$ or $25 \pm 2{ }^{\circ} \mathrm{C}(60 \pm 5 \% \mathrm{RH})$ for 7,14 or 30 days

$n t$ not tested, $P F-S Z-I F X$ PF-06438179/GP1111, $R H$ relative humidity

under extended in-use conditions for intravenous infusion. Potency across batches, concentrations and storage conditions remained within pre-defined acceptance criteria during the 30-day evaluation period.

Finally, we evaluated molecular weight variants, which are also sensitive to changes in storage conditions and formulation and can affect the immunogenicity and potency of medicines [31, 32]. Importantly, SEC and reducing CE-SDS experiments demonstrated no change in charge variants or in molecular weight variants upon dilution of PF-SZ-IFX over the 30-day evaluation period.

Using a similar array of experiments, PF-SZ-IFX was previously shown to be structurally and functionally similar to reference infliximab sourced in the USA and in the EU [7]. A minor limitation of the present study is that only a single infusion bag material was considered; polyethylene infusion bags were selected because they are one of the most commonly used of available options and previous studies have shown compatibility of infliximab across several infusion bag materials, including polyvinylchloride [33]. Strengths of the current study include the use of state-of-the-art analytical techniques and the relevance of the conditions tested to clinical practice. Our study shows results for 0.4 and $4.0 \mathrm{mg} / \mathrm{mL}$ dilutions (i.e. the clinically relevant range) at two different temperature conditions likely to occur before administration to the patient ( 5 and $25{ }^{\circ} \mathrm{C} / 60 \% \mathrm{RH}$ ) for up to 30 days. For the reconstituted solution $(10 \mathrm{mg} / \mathrm{mL})$, results were obtained for up to 30 days at $5{ }^{\circ} \mathrm{C}$ and up to 14 days at $25^{\circ} \mathrm{C}$. The Zessly ${ }^{\circledR}$ summary of product characteristics [10] does not recommend storing diluted solutions for longer than $24 \mathrm{~h}$, so a storage duration of 30 days for the diluted medicine is considered to exceed the duration of storage likely to occur during clinical practice and thus represents a worstcase scenario. No changes affecting dose or efficacy (e.g. resulting from adsorption, aggregation or fragmentation) were observed over the extended 30-day period, confirming that the infliximab biosimilar PF-SZ-IFX is not affected by dilution and formulation conditions required for intravenous infusion and providing reassurance to healthcare professionals that the pharmaceutical safety and efficacy of PF-SZ-IFX is maintained under extended in-use conditions.

\section{Conclusion}

The present physicochemical and biological analyses demonstrated that the Sandoz infliximab biosimilar PF-SZ-IFX is not affected by appropriate reconstitution, dilution and extended storage in infusion bags. Following reconstitution $(10 \mathrm{mg} / \mathrm{mL})$, the stability of PF-SZ-IFX is maintained for 30 days at $5{ }^{\circ} \mathrm{C}$ and for 14 days at $25{ }^{\circ} \mathrm{C} / 60 \% \mathrm{RH}$. Following dilution in polyethylene saline infusion bags $(0.4$ and $4.0 \mathrm{mg} / \mathrm{mL}$ ), the stability of PF-SZ-IFX is maintained for 30 days at $5{ }^{\circ} \mathrm{C}$ and at $25^{\circ} \mathrm{C} / 60 \% \mathrm{RH}$.

Acknowledgements The authors thank M Kivel, F Zemp, J Mavri and M Kocjancic for their contributions to the study and thank S Jalen and M Schiestl for critical review of this manuscript.

\section{Compliance with Ethical Standards}

Conflicts of interest MV is an employee of Lek pharmaceuticals d. d., part of the Novartis group (Menges, Slovenia). MGG is an employee of Novartis Pharma AG (Basel, Switzerland). ER is an employee of Hexal AG, part of the Novartis group (Holzkirchen, Germany).

Funding Professional medical writing and editorial assistance was provided by Laura Maguire, MChem of Spirit and was funded by Hexal AG, a Sandoz company, part of the Novartis group (Holzkirchen, Ger- 
many). The study was funded by and performed on behalf of Hexal AG, a Sandoz company, part of the Novartis group (Holzkirchen, Germany).

Open Access This article is distributed under the terms of the Creative Commons Attribution-NonCommercial 4.0 International License (http://creativecommons.org/licenses/by-nc/4.0/), which permits any noncommercial use, distribution, and reproduction in any medium, provided you give appropriate credit to the original author(s) and the source, provide a link to the Creative Commons license, and indicate if changes were made.

\section{References}

1. European Medicines Agency. Remicade ${ }^{\circledR}$ (infliximab) summary of product characteristics. 2017. https://www.ema.europa.eu/ documents/product-information/remicade-epar-product-infor mation_en.pdf. Accessed 15 Oct 2018.

2. FDA. Remicade ${ }^{\circledR}$ highlights of prescribing information. 2017. https://www.accessdata.fda.gov/drugsatfda_docs/label /2017/761072s000lbl.pdf. Accessed 15 Oct 2018.

3. Greenwald M, Tesser J, Sewell KL. Biosimilars have arrived: rituximab. Arthritis. 2018;2018:6. https://doi.org/10.1155/2018/37628 64.

4. European Medicines Agency. Guideline on similar biological medicinal products. CHMP/437/04 Rev1. 2014. www.ema.europ a.eu/docs/en_GB/document_library/Scientific_guideline/2014/10/ WC500176768.pdf. Accessed 15 Oct 2018.

5. Food and Drug Administration. Scientific consideration in demonstration biosimilarity to a reference product. 2015. www.fda. gov/downloads/drugsguidancecomplianceregulatoryinformation/ guidances/ucm291128.pdf. Accessed 15 Oct 2018.

6. World Health Organization. Guidelines on evaluation of similar biotherapeutic products. 2009. www.who.int/biologicals/areas /biological_therapeutics/biotherapeutics_for_web_22april201 0.pdf. Accessed 15 Oct 2018.

7. Derzi M, Johnson TR, Shoieb AM, Conlon HD, Sharpe P, Saati A, et al. Nonclinical evaluation of PF-06438179: a potential biosimilar to Remicade ${ }^{\circledR}$ (Infliximab). Adv Ther. 2016;33(11):1964-82. https://doi.org/10.1007/s12325-016-0403-9.

8. Palaparthy R, Udata C, Hua SY, Yin D, Cai CH, Salts S, et al. A randomized study comparing the pharmacokinetics of the potential biosimilar PF-06438179/GP1111 with Remicade ${ }^{\circledR}$ (inflixi$\mathrm{mab}$ ) in healthy subjects (REFLECTIONS B537-01). Expert Rev Clin Immunol. 2018;14(4):329-36. https://doi.org/10.1080/17446 66X.2018.1446829.

9. Cohen SB, Alten R, Kameda H, Hala T, Radominski SC, Rehman MI, et al. A randomized controlled trial comparing PF-06438179/GP1111 (an infliximab biosimilar) and infliximab reference product for treatment of moderate to severe active rheumatoid arthritis despite methotrexate therapy. Arthritis Res Ther. 2018;20(1):155. https://doi.org/10.1186/s1307 5-018-1646-4.

10. European Medicines Agency. Zessly summary of product characteristics. 2018. http://www.ema.europa.eu/docs/en_GB/docum ent_library/EPAR_-_Product_Information/human/004647/ WC500249647.pdf. Accessed 15 Oct 2018.

11. PDMA. Infliximab BS for I.V infusion $100 \mathrm{mg}$ [Pfizer] (Infliximab Biosimilar 3) product information. 2018. http://www.pmda.go.jp/ PmdaSearch/iyakuDetail/GeneralList/23994A3F1. Accessed 15 Oct 2018.
12. Food and Drug Administration. IXIFI highlights of prescribing information. 2018. http://www.accessdata.fda.gov. Accessed 15 Oct 2018.

13. International Conference on Harmonization Guideline. International Conference on Harmonization Guideline. Stability testing of new drug substances and products Q1A (R2). 2003. Online referencing, www.ich.org/fileadmin/Public_Web_Site/ICH_Produ cts/Guidelines/Quality/Q1A_R2/Step4/Q1A_R2_Guideline.pdf. Accessed 15 Oct 2018.

14. International Conference on Harmonization Guideline. Stability testing of biotechnological/biological products Q5C. Online referencing. 1995. www.ich.org/fileadmin/Public_Web_Site/ ICH_Products/Guidelines/Quality/Q5C/Step4/Q5C_Guideline. pdf. Accessed 15 Oct 2018.

15. European Medicines Agency. Note for guidance on in-use stability testing of human medicinal products. CPMP/QWP/2934/99. Online referencing. 2001. www.ema.europa.eu/docs/en_GB/ document_library/Scientific_guideline/2009/09/WC50000347 5.pdf. Accessed 15 Oct 2018.

16. European Medicines Agency. Note for guidance on maximum shelf life for sterile products for human use after first opening or following reconstitution. CPMP/QWP/159/96 corr. Online referencing. 1998. www.ema.europa.eu/docs/en_GB/document_libra ry/Scientific_guideline/2009/09/WC500003476.pdf. Accessed 15 Oct 2018.

17. Lamanna WC, Heller K, Schneider D, Guerrasio R, Hampl V, Fritsch $\mathrm{C}$, et al. The in-use stability of the rituximab biosimilar Rixathon ${ }^{\circledR} /$ Riximyo $^{\circledR}$ upon preparation for intravenous infusion. J Oncol Pharm Pract. 2019;25(2):269-78. https://doi. org/10.1177/1078155217731506.

18. European Directorate for the Quality of Medicines. European Pharmacopoeia (9th Edition). Strasbourg: Council of Europe; 2016.

19. United States Pharmacopoeia 41. The National Formulary 36. Rockville, Maryland, USA; 2017.

20. Ministry of Health Labour and Welfare. The Japanese Pharmacopeia. Seventeenth ed. Tokyo: Ministry of Health Labour and Welfare; 2016.

21. United States Pharmacopoeia 41. The National Formulary 36: General Chapters: 788 Particulate matter in injections. Rockville, Maryland, USA: 2017.

22. International Conference on Harmonization Guideline. International Conference on Harmonization Guideline. Test procedures and acceptance criteria for biotechnological/biological products Q6B. Online referencing. 1999. Accessed 15 Feb 2019.

23. International Conference on Harmonization Guideline. Validation of analytical procedures Q2 (R1). Online referencing. 1994. https ://www.ich.org/fileadmin/Public_Web_Site/ICH_Products/Guide lines/Quality/Q2_R1/Step4/Q2_R1_Guideline.pdf. Accessed 15 Feb 2019.

24. Herrmann JE, Collins MF. Quantitation of immunoglobulin adsorption to plastics. J Immunol Methods. 1976;10(4):363-6.

25. Khawli LA, Goswami S, Hutchinson R, Kwong ZW, Yang J, Wang $\mathrm{X}$, et al. Charge variants in $\mathrm{IgG1}$ : isolation, characterization, in vitro binding properties and pharmacokinetics in rats. MAbs. 2010;2(6):613-24. https://doi.org/10.4161/mabs.2.6.13333.

26. Vlasak J, Ionescu R. Heterogeneity of monoclonal antibodies revealed by charge-sensitive methods. Curr Pharm Biotechnol. 2008;9(6):468-81.

27. Jung SK, Lee KH, Jeon JW, Lee JW, Kwon BO, Kim YJ, et al. Physicochemical characterization of Remsima. MAbs. 2014;6(5):1163-77. https://doi.org/10.4161/mabs.32221.

28. Sydow JF, Lipsmeier F, Larraillet V, Hilger M, Mautz B, Molhoj M, et al. Structure-based prediction of asparagine and aspartate degradation sites in antibody variable regions. PLoS One. 
2014;9(6):e100736. https://doi.org/10.1371/journal.pone.01007 36.

29. Pisupati K, Benet A, Tian Y, Okbazghi S, Kang J, Ford M, et al. Biosimilarity under stress: a forced degradation study of Remicade ${ }^{\circledR}$ and Remsima. MAbs. 2017;9(7):1197-209. https:// doi.org/10.1080/19420862.2017.1347741.

30. Liang S, Dai J, Hou S, Su L, Zhang D, Guo H, et al. Structural basis for treating tumor necrosis factor alpha (TNFalpha)-associated diseases with the therapeutic antibody infliximab. J Biol Chem. 2013;288(19):13799-807. https://doi.org/10.1074/jbc. M112.433961.
31. Ratanji KD, Derrick JP, Dearman RJ, Kimber I. Immunogenicity of therapeutic proteins: influence of aggregation. J Immunotoxicol. 2014;11(2):99-109. https://doi.org/10.3109/15476 91X.2013.821564.

32. Vlasak J, Ionescu R. Fragmentation of monoclonal antibodies. MAbs. 2011;3(3):253-63.

33. Maraiki F, Farooq F, Ahmed M. Eliminating the use of intravenous glass bottles using a FOCUS-PDCA model and providing a practical stability reference guide. Int J Pharm Pract. 2016;24(4):271-82. https://doi.org/10.1111/ijpp.12245. 